\title{
Alternative splicing of spleen tyrosine kinase differentially regulates colorectal cancer progression
}

\author{
BEIBEI NI ${ }^{1,2^{*}}$, JUN HU ${ }^{1,2^{*}}$, DIANKE $\mathrm{CHEN}^{2,3}, \mathrm{LI} \mathrm{LI}^{1,2}$, DAICI CHEN $^{1,2}$, JIANPING WANG $^{2,4}$ and LEI WANG ${ }^{2,4}$ \\ ${ }^{1}$ Guangdong Institute of Gastroenterology; ${ }^{2}$ Guangdong Key Laboratory of Colorectal and Pelvic Floor Diseases; \\ Departments of ${ }^{3}$ Medical Oncology and ${ }^{4}$ Colorectal Surgery, The Sixth Affiliated Hospital of Sun Yat-sen University, \\ Guangzhou, Guangdong 510655, P.R. China
}

Received May 18, 2015; Accepted June 21, 2015

DOI: $10.3892 / \mathrm{ol} .2016 .4858$

\begin{abstract}
Spleen tyrosine kinase (SYK) has been reported as a potential tumor suppressor in colorectal cancer (CRC). However, the role of alternative splicing of SYK in carcinogenesis remains unclear. In the present study, SYK isoforms were overexpressed in the human CRC HCT 116 cell line using lentiviral expression vectors to investigate the biological functions of full length SYK [SYK(L)] and short form SYK $[\mathrm{SYK}(\mathrm{S})]$ in CRC. Real-time cellular analysis and the 5-ethynyl-2-deoxyuridine assay were used to detect the effects of SYK(L) and SYK(S) on cell proliferation. Cell cycle progression and migration were assessed via flow cytometry and Transwell assays, respectively. The results revealed that the recombinant lentivirus with SYK(L) overexpression significantly suppressed the proliferation and metastasis of CRC cells, while SYK(S) overexpression did not. In addition, MTS assays demonstrated that $\mathrm{SYK}(\mathrm{L})$ and $\mathrm{SYK}(\mathrm{S})$ increased the cellular sensitivity to 5-fluorouracil (5-FU), suggesting that SYK(L) and 5-FU produce a significant synergistic effect on CRC cell proliferation, while SYK(S) has an effect on modulating CRC 5-FU sensitivity. Furthermore, quantitative polymerase chain reaction results revealed that $\mathrm{SYK}(\mathrm{L})$ was downregulated in $69 \%$ of 26 pairs of CRC and adjacent non-cancerous tissues, whereas $\mathrm{SYK}(\mathrm{S})$ exhibited no significant differences between
\end{abstract}

Correspondence to: Professor Jianping Wang or Professor Lei Wang, Department of Colorectal Surgery, The Sixth Affiliated Hospital of Sun Yat-sen University, 26 Yuancun Erheng Road, Guangzhou, Guangdong 510655, P.R. China

E-mail: wangjply01@sohu.com

E-mail: wang19@mail.sysu.edu.cn

*Contributed equally

Abbreviations: CRC, colorectal cancer; SYK, spleen tyrosine kinase; qPCR, quantitative polymerase chain reaction; GAPDH, glyceraldehyde-3-phosphate dehydrogenase; RTCA, real-time cellular analysis

Key words: SYK, splicing isoform, lentiviral expression vectors, colorectal cancer, 5-FU resistance tumor and normal tissues. Overall, the present data provides evidence that $S Y K(L)$ is a tumor suppressor in CRC, and both SYK(L) and SYK(S) may serve as important predictors in the chemotherapeutic treatment of CRC.

\section{Introduction}

Colorectal cancer (CRC) is one of the most common human cancers worldwide, accounting for 1.2 million novel cases and 600,000 mortalities per year (1). Despite the great progress in current therapeutic options, including surgery and chemoradiotherapy, the incidence and mortality of CRC remains high (2). Novel strategies require investigation not only to enhance the efficacy of currently available agents, but also to identify novel therapeutic targets for CRC patients. Therefore, there are concerted efforts to identify gene expression patterns that may be used for early detection and improved prognosis prediction for CRC.

Spleen tyrosine kinase (SYK) is a $72 \mathrm{kDa}$ non-receptor tyrosine kinase that contains two tandem Src homology 2 domains at the $\mathrm{NH}_{2}$ terminus and a kinase domain at the $\mathrm{COOH}$ terminus (3). It is widely expressed in hematopoietic cells and was hypothesized to be a hematopoietic cell-specific signaling molecule (4). Currently, SYK is considered to be a potential tumor suppressor in breast carcinoma (5), and there are a growing number of studies on SYK in non-hematopoietic tumors (6-8). Several clinical studies have indicated that patients with negative SYK expression have a significantly lower overall survival rate compared with patients with positive SYK expression (9-12). Functional studies have demonstrated that dysregulation of SYK is associated with cancer proliferation and metastasis (13-15). These data implicate SYK as a putative tumor suppressor in cancer. However, Luangdilok et al (16) reported that high expression of SYK was significantly associated with recurrence and poorer survival in squamous cell carcinomas of the head and neck, and these results are consistent with a study concerning nasopharyngeal carcinoma (17). Overall, the present study hypothesizes that SYK has a complex role in multiple cancer types.

SYK has two alternatively spliced isoforms: Full-length [SYK(L)] and short form [SYK(S)] SYK, which lacks a 69-nucleotide exon (Fig. 1A). A previous study by the present authors revealed that SYK(L) was present in the cytoplasm 
and nucleus of breast cancer cells, and suppressed breast cancer cell invasiveness, whereas SYK(S) was located exclusively in the cytoplasm and did not affect breast cancer cell invasion (18). Consistent with these results, recent evidence revealed that differential expression of SYK(L) and SYK(S) may contribute to tumor biology in different ways, and may be clear indicators of prognosis in patients with hepatocellular cancer (19). In addition, Prinos et al (20) have reported that changing the SYK alternative splicing pattern alters cancer cell survival and mitotic progression. On the basis of these data, the present study hypothesizes that SYK alternative splicing isoforms have different functional effects in cancer and act as modulators of cancer.

Hypermethylation of the SYK gene promoter was demonstrated to be associated with a loss of SYK gene expression in a variety of malignant cancers, including breast cancer (21), gastric cancer, nasopharyngeal carcinoma (22) and hepatocellular cancer (23). In CRC, the present authors previously demonstrated that global SYK methylation was an independent prognostic factor for overall survival (12), but the expression and biological functions of alternative splicing SYK isoforms in CRC remain unclear. The present study aimed to investigate the functional impact of SYK(L) and SYK(S) in CRC. The present study evaluated the effect of SYK(L) and SYK(S) on proliferation, metastasis and 5-fluorouracil (5-FU) resistance in CRC cells by overexpressing SYK(L) and SYK(S). In addition, the expression pattern of SYK isoforms was also confirmed in CRC tissues.

\section{Materials and methods}

Clinical samples and cell lines. In total, 26 CRC samples and matched adjacent normal samples were obtained from the Tissue Bank of The Sixth Affiliated Hospital, Sun Yat-sen University (Guangzhou, China) between March 2010 and July 2010. All the samples were obtained with the written informed consent of the patients and were histologically confirmed. The Institutional Review Board of Sun Yat-sen University approved the study.

Seven human CRC cell lines (HCT 116, SW480, RKO, HCT-8, LoVo, HCT-15 and Caco-2) were obtained from Shanghai Cell Collection, Chinese Academy of Science (Shanghai, China). HCT 116, SW480, HCT-8 and HCT-15 were maintained in RPMI-1640 medium (Gibco; Thermo Fisher Scientific, Inc., Waltham, MA, USA), whereas RKO, LoVo and Caco-2 were maintained in Dulbecco's Modified Eagle's Medium (DMEM; Thermo Fisher Scientific, Inc.). All cells were cultured in recommended medium containing $10 \%$ fetal bovine serum (FBS; Thermo Fisher Scientific, Inc.), and maintained at $37^{\circ} \mathrm{C}$ with $5 \% \mathrm{CO}_{2}$ in a humidified incubator. The cell lines were routinely authenticated (once every year) using DNA-fingerprinting analysis, identity verification using short tandem repeat profiling analysis, cell morphology monitoring, growth curve analysis and mycoplasma contamination checks.

Lentiviral vector construction and stable cell lines. The lentiviral plasmid pLVX-EGFP-3FLAG alone (negative control) or with SYK(L) or SYK(S) cDNA were constructed by Sunbio (Shanghai, China). The SYK(L) and SYK(S) gene
cDNA were verified by DNA sequencing and polymerase chain reaction (PCR). The lentiviral vectors expressed green fluorescent protein (GFP); therefore, viral titer was determined by the method of end point dilution by counting the numbers of infected green cells at magnification X100 under a fluorescence microscope (Leica Microsystems GmbH, Wetzlar, Germany). HCT 116 cells, which expressed low detectable SYK(L) and SYK(S), were used to generate stable cells by lentiviral infection, according to the manufacturer's protocol. The same amount of negative control vector was also infected in HCT 116 cells. A total of $12 \mathrm{~h}$ later, the virus-containing medium was replaced with fresh complete medium. Following a $72 \mathrm{~h}$ infection, $1 \mu \mathrm{g} / \mathrm{ml}$ puromycin was added to the culture medium for selection of stable cells. The fluorescence of GFP in stable cell lines was observed by fluorescence microscopy to achieve over 100\% transfection. The expression levels of SYK(L) and SYK(S) in stable cells were evaluated by quantitative (q)PCR.

Reverse transcription-qPCR. Total RNA was extracted from tissues and transfected cells using TRIzol Reagent (Invitrogen $^{\mathrm{TM}}$; Thermo Fisher Scientific, Inc.), according to the manufacturer's protocol. Reverse transcription was performed using $0.5 \mu \mathrm{g}$ RNA and the ReverTra Ace ${ }^{\circledR} \mathrm{qPCR}$ RT kit (cat. no. FSQ-301; Toyobo, Co., Ltd., Osaka, Japan) containing DNase I, according to the manufacturer's protocol. qPCR was conducted on an ABI 7500 system (Applied Biosystems; Thermo Fisher Scientific, Inc.) using the SYBR Green Real-Time PCR Master Mix (Thermo Fisher Scientific, Inc.), with the following cycling conditions: $95^{\circ} \mathrm{C}$ for $10 \mathrm{~min}$; and 40 cycles of $95^{\circ} \mathrm{C}$ for $15 \mathrm{sec} ; 60^{\circ} \mathrm{C}$ for $1 \mathrm{~min}$. qPCR was performed in triplicate, including no-template controls. In each qPCR assay, amplification of the reference gene glyceraldehyde-3-phosphate dehydrogenase (GAPDH) was performed as the internal control. The PCR primers were as follows: SYK(L), forward 5'-GCTCTGGCAGCTAGTCGA -3 ' and reverse 5'-GCTTTGGGAAGGAGTATGA-3'; SYK(S), forward 5'-AAAGCAGATGGTTTGTTAAGAGTTC-3' and reverse 5'-CTTGGGCAGGGGAGGACGCAGGATG-3'; GAPDH, forward 5'-GACAGTCAGCCGCATCTTCTT-3' and reverse 5'-AATCCGTTGACTCCGACCTTC-3'. The relative amount of SYK(L) and SYK(S) to GAPDH were calculated using the quantitative cycle threshold $\left(2^{-\Delta \Delta} \mathrm{Cq}\right)$ method (24).

Western blot. The cells were lysed in RIPA lysis buffer (DingGuo Biotechnology, Co., Ltd., Beijing, China) supplemented with phenylmethanesulfonyl fluoride (DingGuo Biotechnology, Co., Ltd.) and centrifuged at $10,000 \mathrm{x}$ g for $5 \mathrm{~min}$ at $4^{\circ} \mathrm{C}$. The protein samples were separated by $8 \%$ sodium dodecyl sulfate-polyacrylamide gel electrophoresis and the products were electrotransferred to nitrocellulose filter membrane (GE Healthcare Life Scientific, Chalfont, UK). Rabbit anti-SYK polyclonal antibody (cat. no. sc-1077; dilution, 1:700; Santa Cruz Biotechnology, Inc., Dallas, TX, USA) and mouse anti-GAPDH monoclonal antibody (cat. no. 60004-1-lg; dilution, 1:4,000; Proteintech, Rosemont, IL, USA) were used for immunodetection. Following incubations with primary antibodies at $4^{\circ} \mathrm{C}$ overnight, IRDye ${ }^{\circledR} 800 \mathrm{CW}$-conjugated goat anti-rabbit and IRDye ${ }^{\circledR} 680 \mathrm{RD}$-conjugated goat anti-mouse secondary antibodies (cat. nos. P/N 925-32211 and P/N 925-68070, respectively; dilution, 1:10,000; LI-COR Biosciences, Lincoln, 
NE, USA) were incubated with the membranes for $1 \mathrm{~h}$ at room temperature. Proteins were detected using the Odyssey ${ }^{\circledR}$ CLx Imaging System (LI-COR Biosciences), and the band intensities were analyzed using the Odyssey Application 3.0 software (LI-COR Biosciences).

Cell proliferation assays. Cell proliferation was measured using the xCELLigence Real Time Cell Analyzer (RTCA) (ACEA Biosciences, San Diego, CA, USA). Briefly, cells were seeded in E-plates at a density of 6,000 cells per well and incubated at $37^{\circ} \mathrm{C}$ and $5 \% \mathrm{CO}_{2}$. The cell growth curves were automatically monitored and recorded every $15 \mathrm{~min}$ for a total of $120 \mathrm{~h}$. Cells adhered to the bottom of each well and cell index values were measured by monitoring cells that covered the surface of the sensor. Experiments were repeated independently three times.

Cell proliferation was also measured by 5-ethynyl-2-deoxyuridine (EdU) assay using an EdU Assay kit (Guangzhou Ribobio, Co., Ltd., Guangzhou, China), according to the manufacturer's protocol. Briefly, cells were cultured in 96-well plates and then exposed to $50 \mathrm{mM} \mathrm{EdU}$ for $2 \mathrm{~h}$ at $37^{\circ} \mathrm{C}$. Subsequently, the cells were fixed with $4 \%$ formaldehyde for $30 \mathrm{~min}$ at room temperature. Cells were washed with phosphate-buffered saline (PBS) and incubated with $100 \mu 1$ 1X Apollo ${ }^{\circledR}$ reaction cocktail for $30 \mathrm{~min}$. The DNA contents of cells were stained with $100 \mu 1$ Hoechst 33342 for $30 \mathrm{~min}$ and viewed under a fluorescent microscope.

Cell cycle assay. Cells were stained using Cell Cycle Staining kit (Lianke Bio, Hangzhou, China) and analyzed by flow cytometry. In brief, a pellet of $\sim 2 \times 10^{5}$ cells was formed by centrifugation and the cells were washed with PBS. Subsequently, $1 \mathrm{ml}$ cold $75 \%$ ethanol was added to the cells at $-20^{\circ} \mathrm{C}$ overnight. Subsequently, the cells were rehydrated with PBS for $15 \mathrm{~min}$ and incubated with $1 \mathrm{ml}$ DNA staining solution at room temperature. The cells were examined using a flow cytometer (BD Biosciences, Franklin Lakes, NJ, USA). The results were analyzed using the ModFit version 3.0 software (Verity Software House, Topsham, ME, USA).

Cell migration and invasion assays. Cell invasion assays were performed using a 24-multiwell insert plate with an $8.0 \mu \mathrm{m}$ pore size membrane chamber (BD Biosciences) containing a Matrigel-coated membrane. Cells were suspended in serum-free DMEM and added to apical chambers. Cells at a density of 40,000 cells/100 $\mu \mathrm{l}$ of DMEM media were placed into the upper chamber of the Transwell plate and $700 \mu 1$ DMEM medium containing $20 \%$ FBS was added to the basal chambers. Following a $48 \mathrm{~h}$ incubation, the cells in the basal chamber were stained with 4',6-diamidino-2-phenylindole, dihydrochloride (Roche Diagnostics GmbH, Mannheim, Germany) and counted using a fluorescence microscope. The cell migration assay was performed in the same way without Matrigel. Three independent experiments were conducted.

Cell viability assay. 5-FU resistance was measured by a 3-(4,5-dimethylthiazol-2-yl)-5-(3-carboxymethoxyphenyl)-2(4-sulfophenyl)-2H-tetrazolium (MTS) assay (Promega, Madison, WI, USA), which assesses the reduction of MTS to formazan in viable cells. Briefly, cells were seeded in 96-well plates at a density of 5,000 cells $/ 100 \mu \mathrm{l} /$ well. Subsequent to $24 \mathrm{~h}$, cells were treated with various concentrations of 5-FU $(0,0.1,1.0,10.0,20.0,50.0,100.0$ and $200.0 \mu \mathrm{M})$. Following a $72 \mathrm{~h}$ incubation, $20 \mu \mathrm{l}$ of the combined MTS/phenazine methosulfate solution was added to the medium and the mixture was incubated at $37^{\circ} \mathrm{C}$ for $1.5 \mathrm{~h}$. The absorbance was measured at $490 \mathrm{~nm}$ using Varioskan ${ }^{\mathrm{TM}}$ Flash multimode reader (Thermo Fisher Scientific, Inc.).

Statistical analysis. Statistical analysis was performed with SPSS version 16.0 software (SPSS, Inc., Chicago, IL, USA). Data are presented as the mean \pm standard deviation. Differences between groups were compared using paired or unpaired Student's t-tests or the Mann-Whitney U test. $\mathrm{P} \leq 0.05$ was considered to indicate a statistically significant difference.

\section{Results}

Stably transfected cells overexpress SYK isoforms. To examine the biological impact of SYK splice isoforms in CRC cells, the present study first identified the expression of SYK splice isoforms SYK(L) and SYK(S) in 7 CRC cell lines (HCT 116, SW480, $\mathrm{Caco}_{2}$, LoVo, RKO, HCT-8 and HCT-15) using western blot analysis and qPCR (Fig. 1B). Western blot analysis revealed that $\mathrm{SYK}(\mathrm{L})$ was highly expressed in SW480, LoVo, HCT-15 and RKO cell lines, with low or no expression in HCT 116, $\mathrm{Caco}_{2}$ and HCT-8. SYK(S) protein was detected in SW480 and $\mathrm{Caco}_{2}$ cells, and was almost undetectable in other cell lines. Subsequently, mRNA levels of SYK(L) and SYK(S) in the 7 cell lines were identified by qPCR. The alterations in the mRNA expression of SYK isoforms between cell lines was consistent with the protein levels in these cells.

HCT 116 cells were selected as the target cells for transfection with the pLVX-EGFP-3FLAG-SYK(L)/SYK(S) recombinant lentiviral vector or empty vector. Although the expression of SYK(L) and SYK(S) was negligible in both HCT 116 cells and HCT-8 cells, the biological functions of these isoforms of SYK were more obvious and stable in HCT 116 cells, as compared with HCT-8 cells; thus HCT 116 cells were selected for further studies. Results of this transfection revealed that the transfected HCT 116 cells expressed GFP following transfection and green fluorescence was detected using a fluorescent microscope (Fig. 1C). qPCR demonstrated that the relative expression levels of SYK(L) and SYK(S) were markedly increased in the SYK(L) and SYK(S) overexpression cells compared with those in the negative control group (Fig. 1D).

$S Y K(L)$, but not $S Y K(S)$, suppresses the proliferation of $C R C$ cells via cell cycle arrest. To investigate the potential effects of SYK isoforms in CRC cells in vitro, transfected HCT 116 cells underwent RTCA and EdU assay. As shown in Fig. 2A, the RTCA revealed that the SYK(L) overexpression group clearly suppressed cell proliferation compared with the negative control group $(\mathrm{P}=0.004)$, whereas the $\mathrm{SYK}(\mathrm{S})$ overexpression group did not significantly affect HCT 116 cell proliferation $(\mathrm{P}=0.063)$. These findings were further confirmed by an EdU assay (Fig. 2B); the percentage of EdU-positive cells was 
A

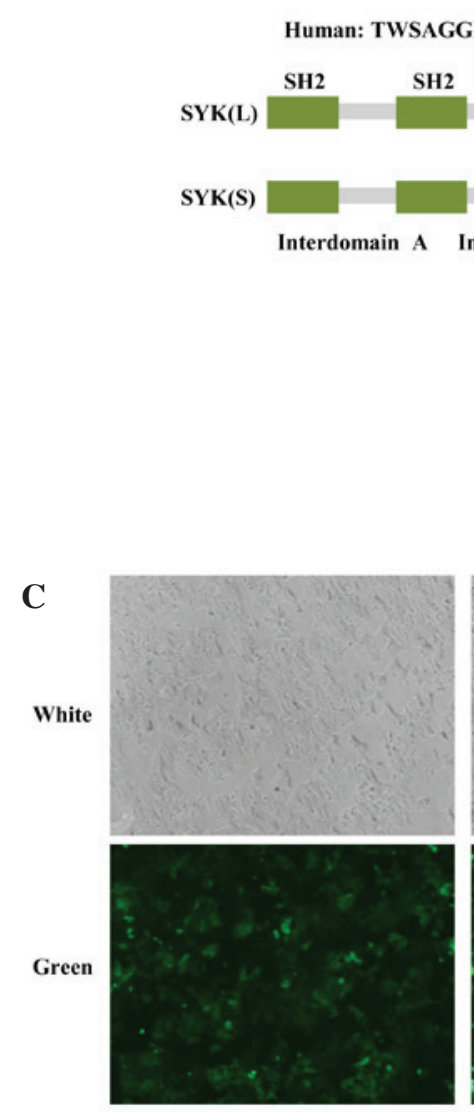

SYK(L)
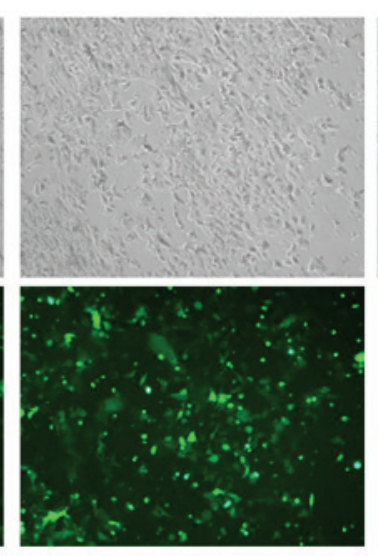

SYK(S)
B
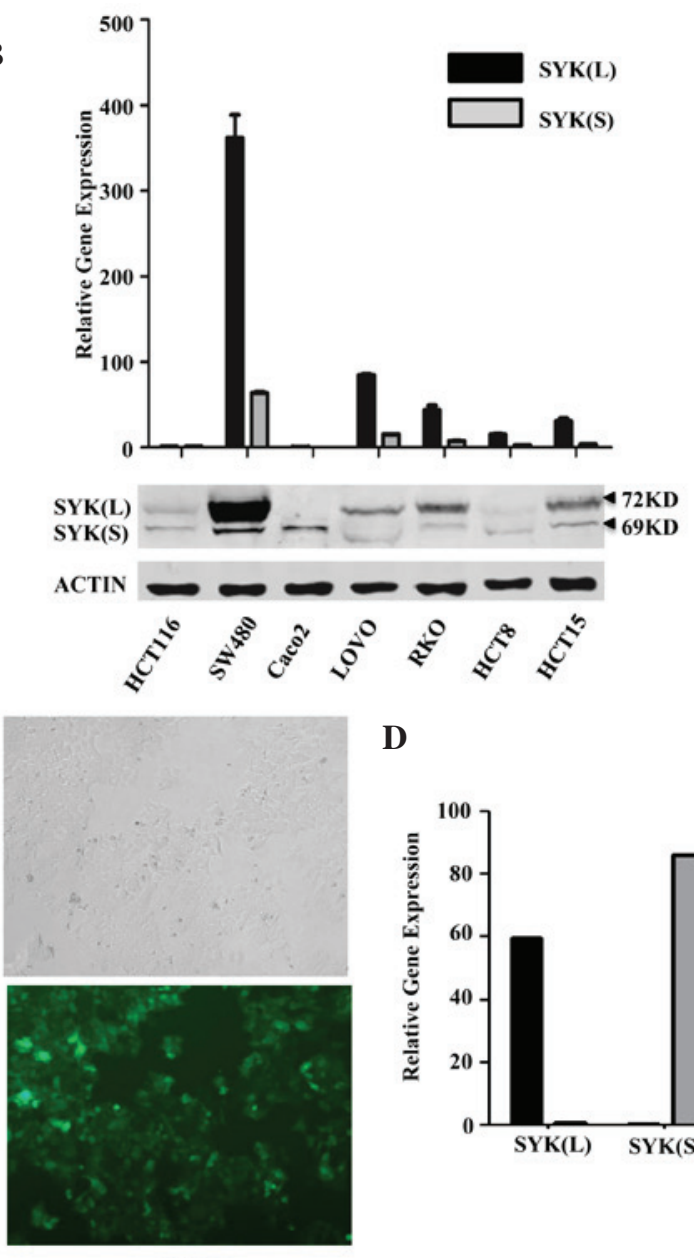

D

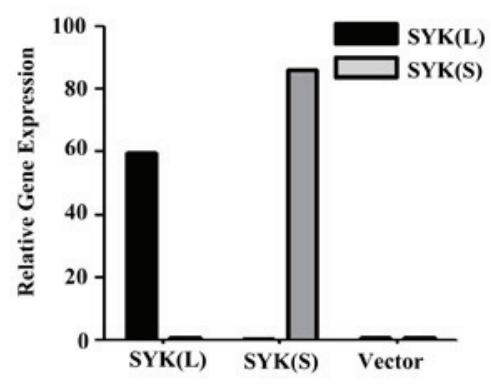

Figure 1. Human CRC HCT 116 cells transfected with recombinant lentiviral vectors with SYK(L) or SYK(S). (A) Domain structure of SYK(L) protein and its alternative splicing variant SYK(S). (B) Analysis of SYK(L) and SYK(S) expression in 7 CRC cell lines by qPCR and western blot analysis. (C) Identification of positive clones with recombinant lentiviral vectors with SYK(L) or SYK(S). (D) Detection of SYK(L) and SYK(S) expression levels in HCT 116 cells by qPCR. SYK, spleen tyrosine kinase; CRC, colorectal cancer; SYK(L), SYK full length; SYK(S), SYK short form; SH2, Src homology 2.

significantly lower in cells expressing $\mathrm{SYK}(\mathrm{L})(\mathrm{P}=0.020)$. However, there was no significant difference between SYK(S) overexpression group and the negative control group $(\mathrm{P}=0.054)$. Overall, these findings suggest that SYK(L), and not SYK(S), suppress the cell proliferation capability of HCT 116 cells.

Fluorescence-activated cell sorting analysis was conducted to analyze the effect of the SYK splice isoforms on cell cycle progression. As shown in Fig. 2C, the percentage of cells in the G2M-phase of the SYK(L) overexpression group was significantly increased compared with those of the negative control group $(\mathrm{P}=0.018)$, whereas $\mathrm{SYK}(\mathrm{S})$ overexpression did not significantly alter the percentage of cells in the $\mathrm{G} 2 \mathrm{M}$ phase $(\mathrm{P}=0.617)$. Collectively, these results suggest that SYK(L) inhibits the proliferation of HCT 116 cells via inducing G2M phase arrest.

$S Y K(L)$ not $S Y K(S)$ suppress metastasis in CRC cells. Subsequently, whether SYK(L) and SYK(S) had an effect on the migration and invasion ability of HCT 116 cells was analyzed by Transwell assay. Cells on the lower side of the Transwell membrane were the migrating/invading cells. Representative photomicrographs of Transwell assays were obtained at magnification x100 (Fig. 3A). Subsequently, the number of cells were counted in nine independent visual fields under the microscope (magnification, x200) from three independent experiments. Overexpression of SYK(L) significantly decreased the migration and invasion capacity by $84 \%(\mathrm{P}=0.003)$ and $61 \%$ $(\mathrm{P}=0.044)$, respectively, in HCT 116 cells compared with negative control cells (Fig. 3A). However, overexpression of SYK(S) did not significantly affect the motility $(\mathrm{P}=0.325)$ or invasive ability $(\mathrm{P}=0.271)$ of the cells. Therefore, the results revealed that $\mathrm{SYK}(\mathrm{L})$, and not $\mathrm{SYK}(\mathrm{S})$, is involved in CRC cell migration and invasion.

SYK $(L)$ and SYK $(S)$ suppress the 5-FU resistance of CRC cells. Since 5-FU is one of the most commonly used anticancer drugs in CRC $(25,26)$, cell viability following 5-FU treatment of cells overexpressing SYK(L) or SYK(S), or negative control cells, was assessed by the present study in vitro. HCT 116 cells were treated with $0,0.1,1.0,10.0,20.0,50.0,100.0$ and $200.0 \mu \mathrm{M}$ 5 -FU and cell viability was measured by MTS assay. The $\mathrm{IC}_{50}$ values for the $\mathrm{SYK}(\mathrm{L})$ and $\mathrm{SYK}(\mathrm{S})$ overexpression groups and the negative control group were $9.241 \pm 1.552,9.305 \pm 0.341$ and $26.996 \pm 1.639 \mu \mathrm{M}$, respectively (Fig. 3B). The overexpression of SYK(L) cells exhibited a significant reduction in cell viability compared to the negative control group ( $\mathrm{P}=0.026)$. Notably, the overexpression of SYK(S) in HCT 116 cells exhibited a 

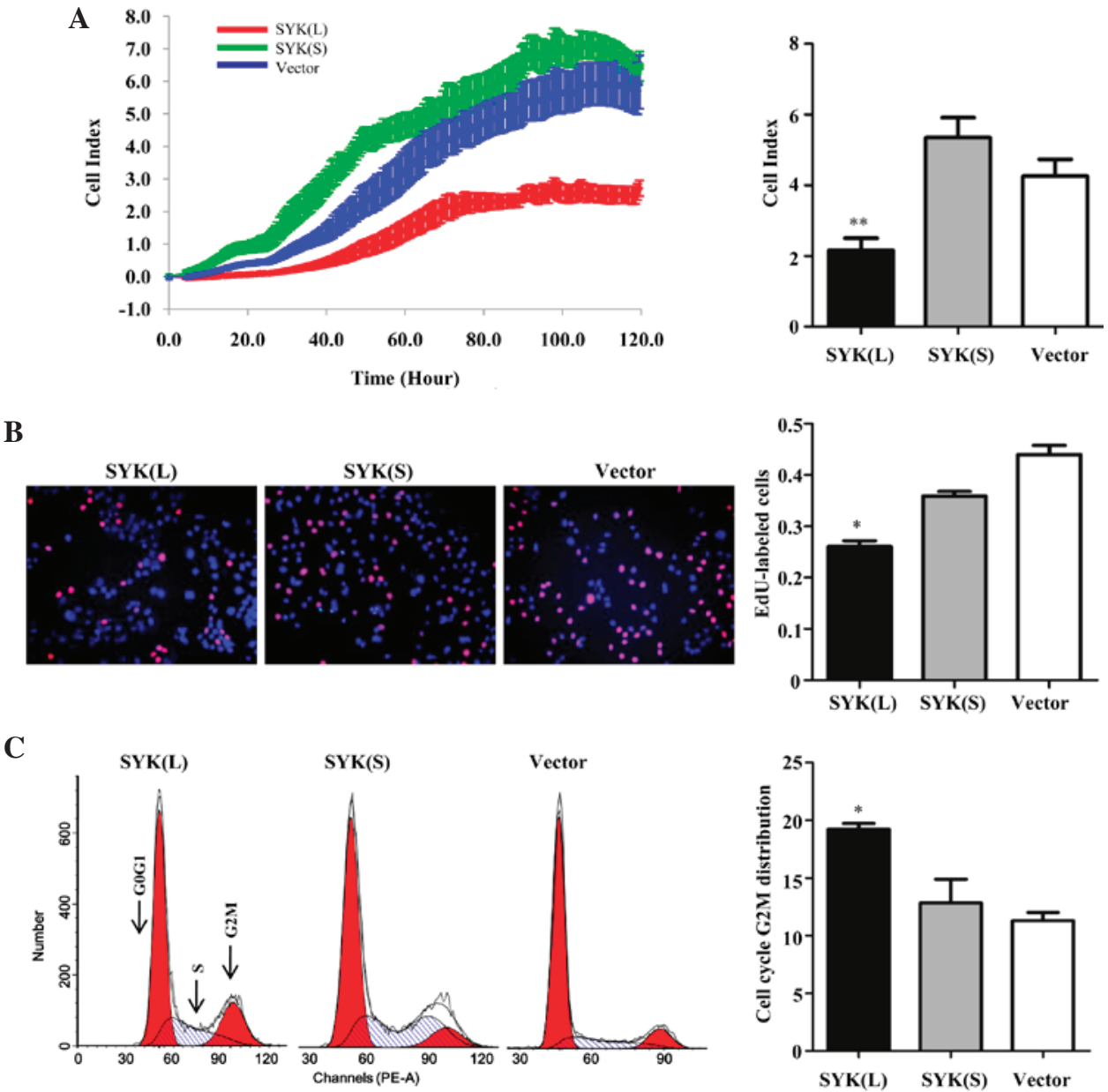

Figure 2. Effects of ectopic SYK(L) and SYK(S) expression on CRC tumor growth. (A) Proliferation curves of human CRC HCT 116 cells analyzed by xCelligence real time cellular analysis following transfection with recombinant lentivirus vector with SYK(L) or SYK(S). Red, green and blue indicate the SYK(L) overexpression, SYK(S) overexpression and negative control groups, respectively. (B) Cells transfected with recombinant lentivirus vector with SYK(L) or SYK(S) were EdU-labeled and compared with control cells. Red and blue indicate EdU- and Hoechst 33342-labeled cells, respectively. (C) Cell cycle progression analysis in HCT 116 cells following transfection with recombinant lentivirus vector with SYK(L) or SYK(S). Data are presented as the mean \pm standard deviation. "P<0.05, " $\mathrm{P}<0.01$ vs. empty vector transfected cells. SYK, spleen tyrosine kinase; SYK(L), SYK full length; SYK(S), SYK short form; EdU, 5-ethynyl-2-deoxyuridine; CRC, colorectal cancer.

higher sensitivity to 5-FU compared with the negative control group $(\mathrm{P}=0.005)$. These results suggest that overexpression of SYK(L) exerts a strong synergistic effect with 5-FU on the growth of HCT 116 cells, and overexpression of SYK(S) has an effect on modulating CRC 5-FU sensitivity.

Expression of SYK splice isoforms in CRC tissues. To investigate the expression pattern of SYK splice isoforms SYK(L) and $\mathrm{SYK}(\mathrm{S})$ in CRC patients, the mRNA levels of SYK(L) and SYK(S) were assessed in 26 tumor tissues and paired normal tissues using qPCR. As shown in Fig. 4, the levels of SYK(L) were significantly lower in tumor tissues compared with normal tissues $(\mathrm{P}=0.049)$. However, the levels of SYK(S) had no significant differences between tumor tissues and normal tissues $(\mathrm{P}=0.403)$. Therefore, $\mathrm{SYK}(\mathrm{L})$ may be recognized as a potential tumor suppressor in CRC.

\section{Discussion}

Alternative splicing allows pre-mRNA to be spliced into multiple mRNAs and protein variants, which exhibit distinct functions (27). Splice variants have been identified for a series of functionally associated cancer genes (28-30), suggesting that alterations in alternative splicing isoforms may contribution to cancer biology or carcinogenesis. A previous study performed a high-throughput survey of 600 alternative splicing cancer-associated genes in breast cancer, and revealed that 41 ductal breast cancer-specific markers may be functionally associated with tumor biology (31). Miura et al (32) reported that systematic dysregulation of alternative spliced isoforms, including vascular endothelial growth factor A and uridine-5'-diphosphate glucuronosyltransferase family 1 , are crucial for the development of novel therapeutic targets for CRC. Splice isoforms often have distinct functions and even exhibit antagonistic properties of oncogenes and tumor suppressors. Thus, it is important to understand how alternative splicing isoforms contribute to the progression of cancer.

SYK is indispensable in inflammatory responses (33) and has been identified as an oncogenic driver in a broad spectrum of hematological malignancies (34). Accumulating evidence revealed that SYK is also required for non-hematopoietic tumors (35-39), including colorectal, breast and hepatocellular 
A

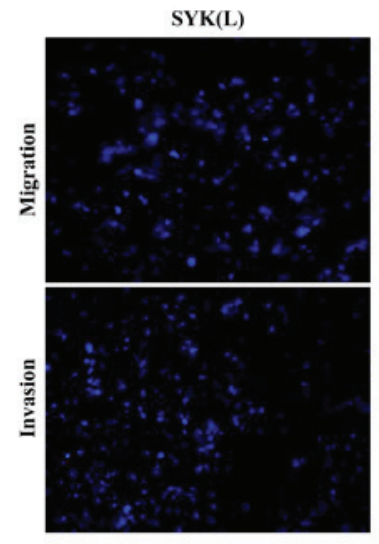

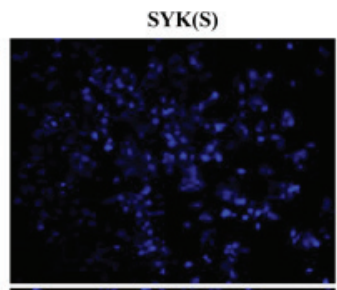

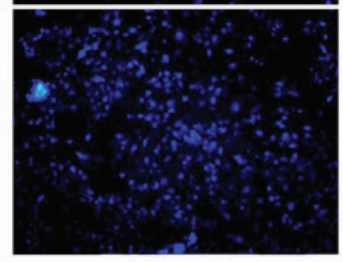

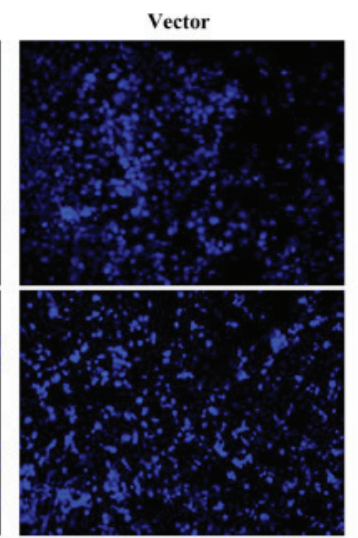

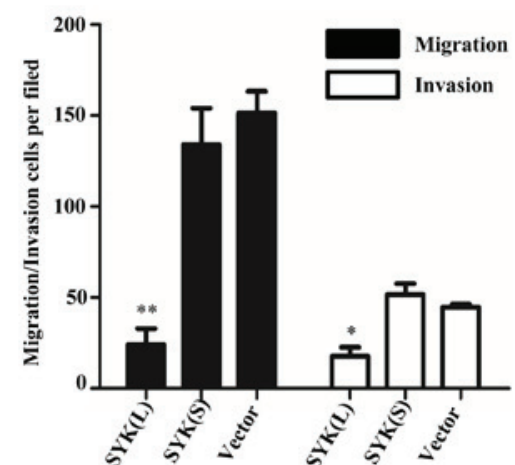

B
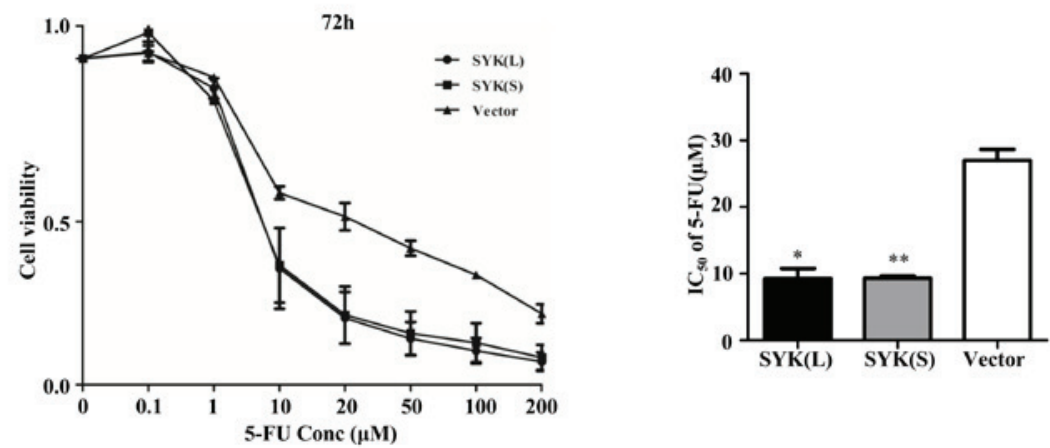

Figure 3. Effects of ectopic SYK(L) and SYK(S) expression on CRC tumor metastasis and 5-FU resistance. (A) Cell migration and invasion assays in human CRC HCT 116 cells following transfection with recombinant lentivirus vector with SYK(L) or SYK(S). Representative microscopy images of invaded and migrated cells of three independent experiments. (B) Effects of SYK(L) and SYK(S) on 5-FU resistance in CRC cells by 3-(4,5-dimethylthiazol-2-yl)-5-( 3-carboxymethoxyphenyl)-2-(4-sulfophenyl)-2H-tetrazolium cell viability assay. 5-FU half maximal inhibitory concentrations were calculated from three independent experiments performed in quadruplicate. Data are presented as the mean \pm standard deviation. ${ }^{*} \mathrm{P}<0.05$, ${ }^{* *} \mathrm{P}<0.01 \mathrm{vs}$. empty vector transfected cells. SYK, spleen tyrosine kinase; SYK(L), SYK full length; SYK(S), SYK short form; 5-FU, .5-fluorouracil; CRC, colorectal cancer.

cancer. In contrast to the possible tumor-promoting functions for SYK in hematological tumors (4), global SYK inversely inhibits tumor progression in the majority of non-hematopoietic tumors $(6,8)$. The present authors previously demonstrated that an aberrant expression of SYK(L) and SYK(S) exhibits a differential phenotypic response, due to their different subcellular localization in breast cancer $(18)$. Hong et al $(9,19)$ reported that phosphorylation of Ser295 in SYK(L) was regulated by checkpoint kinase 1, and observed that SYK(L) and SYK(S) have opposing functions in hepatocellular carcinoma. The present study investigated the biological functions of the two isoforms SYK(L) and SYK(S) in CRC. In agreement with the results in breast cancer and hepatocellular cancer, the present study demonstrated that overexpression of SYK(L) could suppress the proliferation and invasion of CRC cells. However, unlike the results with hepatocellular cancer, the present study revealed that overexpression of SYK(S) resulted in no difference in the capability of cells to proliferate and metastasize compared with negative control in CRC. Furthermore, the present results revealed that $\mathrm{SYK}(\mathrm{L})$ was downregulated in $69 \%$ of 26 pairs of CRC tissues, and the expression of SYK(S) had no significant difference between CRC tissues and adjacent normal tissues. The differences in SYK behavior in various cancer types has been reported to be due to the differences in the contribution of SYK to tumor biology $(19,20)$. The present results indicate that splice isoforms of the SYK gene have complex mechanisms in cancer.
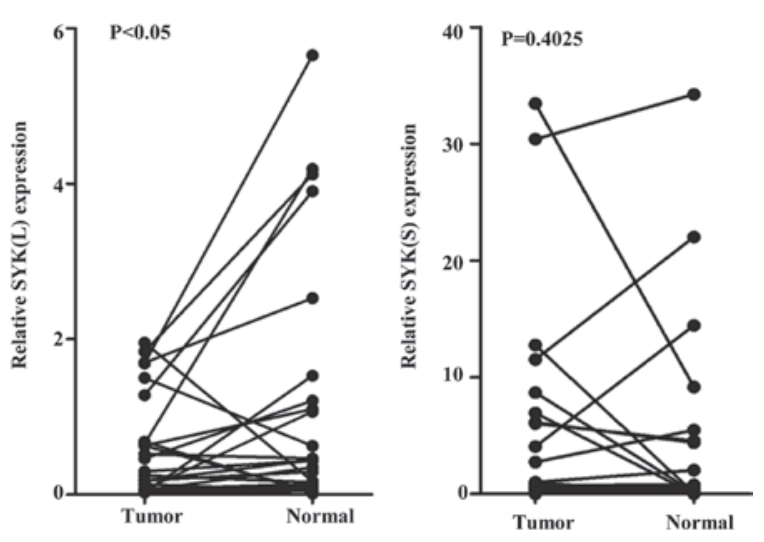

Figure 4. Expression of SYK isoforms in CRC tissues. SYK(L) and SYK(S) mRNA expression was detected by quantitative polymerase chain reaction in 26 pairs of CRC tissues and matched adjacent non-cancerous tissues. Glyceraldehyde 3-phosphate dehydrogenase was used as an endogenous control. SYK, spleen tyrosine kinase; SYK(L), SYK full length; SYK(S), SYK short form.

5-FU is a commonly used chemotherapeutic agent in CRC; however, drug resistance is a major reason for the failure of this drug in the treatment of CRC patients. The present study revealed that SYK(L) and SYK(S) increased the sensitivity of CRC cells to 5-FU. Although overexpression of SYK(S) did not alter cell proliferation and metastasis under normal growth conditions, unlike SYK(L), it decreased cell viability 
following treatment of CRC cells with 5-FU. These findings suggest that SYK(L) and SYK(S) have clinical value in 5-FU cancer therapeutics. Further studies are required to investigate the potential mechanism of SYK in 5-FU resistance in CRC cells.

In conclusion, the present results demonstrate that $\mathrm{SYK}(\mathrm{L})$ and SYK(S) have different expression and biological functions in CRC. SYK(L) not only acted as a tumor suppressor in CRC, but also had effects on chemotherapy resistance. Similarly, SYK $(\mathrm{S})$ is important in chemotherapeutic treatment of CRC. The present study concerning the impact of alternative splicing in cancer biology provides important insights in identifying potential biomarkers and therapeutic targets for CRC.

\section{Acknowledgements}

The present study was supported by the National Key Clinical Discipline, National Natural Scientific Foundation of China (Beijing, China; grant no., 81372566), Science and Technology Plan Project of Guangzhou (Guangzhou, China; grant no., 2013J4500045) and Doctoral Fund of Ministry of Education of China (Beijing, China; grant no., 20120171110095).

\section{References}

1. Brenner H, Kloor M and Pox CP: Colorectal cancer. Lancet 383: 1490-1502, 2014.

2. Guo P, Huang ZL, Yu P and Li K: Trends in cancer mortality in China: An update. Ann Oncol 23: 2755-2762, 2012.

3. Grädler U, Schwarz D, Dresing V, Musil D, Bomke J, Frech M, Greiner H, Jäkel S, Rysiok T, Müller-Pompalla D and Wegener A: Structural and biophysical characterization of the Syk activation switch. J Mol Biol 425: 309-333, 2013.

4. Coopman PJ and Mueller SC: The Syk tyrosine kinase: A new negative regulator in tumor growth and progression. Cancer Lett 241: 159-173, 2006.

5. Coopman PJ, Do MT, Barth M, Bowden ET, Hayes AJ, Basyuk E, Blancato JK, Vezza PR, McLeskey SW, Mangeat PH and Mueller SC: The Syk tyrosine kinase suppresses malignant growth of human breast cancer cells. Nature 406: 742-747, 2000

6. Wang L, Devarajan E, He J, Reddy SP and Dai JL: Transcription repressor activity of spleen tyrosine kinase mediates breast tumor suppression. Cancer Res 65: 10289-10297, 2005.

7. Zhang X, Shrikhande U, Alicie BM, Zhou Q and Geahlen RL: Role of the protein tyrosine kinase Syk in regulating cell-cel adhesion and motility in breast cancer cells. Mol Cancer Res 7: 634-644, 2009.

8. Ogane S, Onda T, Takano N, Yajima T, Uchiyama $T$ and Shibahara T: Spleen tyrosine kinase as a novel candidate tumor suppressor gene for human oral squamous cell carcinoma. Int J Cancer 124: 2651-2657, 2009.

9. Hong J, Hu K, Yuan Y, Sang Y, Bu Q, Chen G, Yang L, Li B, Huang P, Chen D, et al: CHK1 targets spleen tyrosine kinase (L) for proteolysis in hepatocellular carcinoma. J Clin Invest 122: 2165-2175, 2012.

10. Nakashima $H$, Natsugoe $S$, Ishigami $S$, Okumura $H$, Matsumoto M, Hokita S and Aikou T: Clinical significance of nuclear expression of spleen tyrosine kinase (Syk) in gastric cancer. Cancer Lett 236: 89-94, 2006.

11. Peng C, Sun Q, Hao Y, Cong B, Zhao Y and Zhao X: Syk is low-expressed in non-small-cell lung cancer and inversely correlates with patient's survival. Acta Bioch Biophys Sin (Shanghai) 45: 149-151, 2013.

12. Yang Z, Huo L, Chen H, Ni B, Xiang J, Kang L, Wang L, Peng J, Yuan Y and Wang J: Hypermethylation and prognostic implication of Syk gene in human colorectal cancer. Med Oncol 30, 2013.

13. Layton T, Stalens C, Gunderson F, Goodison S and Sillett S: Syk tyrosine kinase acts as a pancreatic adenocarcinoma tumor suppressor by regulating cellular growth and invasion. Am J Pathol 175: 2625-2636, 2009.
14. Yuan YF, Wang JP, Li JP, Wang L, Li M, Yang Z, Zhang C and Dai JL: Frequent epigenetic inactivation of spleen tyrosine kinase gene in human hepatocellular carcinoma. Clin Cancer Res 12: 6687-6695, 2006.

15. Peng CL, Zhang Y, Sun QF, Zhao YP, Hao YT, Zhao XG and Cong B: Inhibitory effects of Syk transfection on lung cancer cell invasion. Asian Pac J Cancer Prev 14: 3001-3003, 2013.

16. Luangdilok S, Box C, Patterson L, Court W, Harrington K, Pitkin L, Rhŷs-Evans P, O-charoenrat P and Eccles S: Syk tyrosine kinase is linked to cell motility and progression in squamous cell carcinomas of the head and neck. Cancer Res 67: 7907-7916, 2007.

17. Du ZM, Kou CW, Wang HY, Huang MY, Liao DZ, Hu CF, Chen J, Yan LX, Hu LF, Ernberg I, et al: Clinical significance of elevated spleen tyrosine kinase expression in nasopharyngeal carcinoma. Head Neck 34: 1456-1464, 2012.

18. Wang L, Duke L, Zhang PS, Arlinghaus RB, Symmans WF, Sahin A, Mendez R and Dai JL: Alternative splicing disrupts a nuclear localization signal in spleen tyrosine kinase that is required for invasion suppression in breast cancer. Cancer Res 63: 4724-4730, 2003.

19. Hong J, Yuan YF, Wang JP, Liao Y, Zou R, Zhu C, Li B, Liang Y, Huang P, Wang Z, et al: Expression of variant isoforms of the tyrosine kinase SYK determines the prognosis of hepatocellular carcinoma. Cancer Res 74: 1845-1856, 2014.

20. Prinos P, Garneau D, Lucier JF, Gendron D, Couture S, Boivin M, Brosseau JP, Lapointe E, Thibault P, et al: Alternative splicing of SYK regulates mitosis and cell survival. Nat Struct Mol Biol 18: 673-679, 2011.

21. Yuan Y, Mendez R, Sahin A and Dai JL: Hypermethylation leads to silencing of the SYK gene in human breast cancer. Cancer Res 61: 5558-5561, 2001.

22. Yan C, Liu C, Jin Q, Li Z, Tao B and Cai Z: The promoter methylation of the Syk gene in nasopharyngeal carcinoma cell lines. Oncol Lett 4: 505-508, 2012.

23. Shin SH, Lee KH, Kim BH, Lee S, Lee HS, Jang JJ and Kang GH: Downregulation of spleen tyrosine kinase in hepatocellular carcinoma by promoter $\mathrm{CpG}$ island hypermethylation and its potential role in carcinogenesis. Lab Invest 94: 1396-1405, 2014.

24. Livak KJ and Schmittgen TD: Analysis of relative gene expression data using real-time quantitative PCR and the 2(-Delta Delta C(T)) Method. Methods 25: 402-408, 2001.

25. Davies JM and Goldberg RM: Treatment of metastatic colorectal cancer. Semin Oncol 38: 552-560, 2011.

26. Shen L, Yu M, Xu X, Gao L, Ni J, Luo Z and Wu S: Knockdown of $\beta 3 \mathrm{GnT} 8$ reverses 5 -fluorouracil resistance in human colorectal cancer cells via inhibition the biosynthesis of polylactosamine-type N-glycans. Int J Oncol 45: 2560-2568, 2014.

27. Ladomery M: Aberrant alternative splicing is another hallmark of cancer. Int J Cell Biol 2013: 463786, 2013.

28. Hatakeyama K, Wakabayashi-Nakao K, Ohshima K, Sakura N, Yamaguchi K and Mochizuki T: Novel protein isoforms of carcinoembryonic antigen are secreted from pancreatic, gastric and colorectal cancer cells. BMC Res Notes 6: 381, 2013.

29. Kahkhaie KR, Moaven O, Abbaszadegan MR, Montazer M and Gholamin M: Specific MUC1 splice variants are correlated with tumor progression in esophageal cancer. World J Surg 38: 2052-2057, 2014

30. Jacob AG, O'Brien D, Singh RK, Comiskey DF Jr, Littleton RM, Mohammad F, Gladman JT, Widmann MC, Jeyaraj SC, Bolinger C, et al: Stress-induced isoforms of MDM2 and MDM4 correlate with high-grade disease and an altered splicing network in pediatric rhabdomyosarcoma. Neoplasia 15: 1049-1063, 2013

31. Venables JP, Klinck R, Bramard A, Inkel L, Dufresne-Martin G, Koh C, Gervais-Bird J, Lapointe E, Froehlich U, Durand M, et al: Identification of alternative splicing markers for breast cancer. Cancer Res 68: 9525-9531, 2008.

32. Miura K, Fujibuchi W and Unno M: Splice isoforms as therapeutic targets for colorectal cancer. Carcinogenesis 33: 2311-2319, 2012.

33. Geahlen RL: Getting Syk: Spleen tyrosine kinase as a therapeutic target. Trends Pharmacol Sci 35: 414-422, 2014.

34. Puissant A, Fenouille N, Alexe G, Pikman Y, Bassil CF, Mehta S, Du J, Kazi JU, Luciano F, Rönnstrand L, et al: SYK is a critical regulator of FLT3 in acute myeloid leukemia. Cancer Cell 25: 226-242, 2014.

35. Shakeel S, Mahjabeen I, Kayani MA and Faryal R: Association of SYK genetic variations with breast cancer pathogenesis. Asian Pac J Cancer Prev 14: 3309-3314, 2013. 
36. Hoellenriegel J, Coffey GP, Sinha U, Pandey A, Sivina M, Ferrajoli A, Ravandi F, Wierda WG, O'Brien S, Keating MJ and Burger JA: Selective, novel spleen tyrosine kinase (Syk) inhibitors suppress chronic lymphocytic leukemia B-cell activation and migration. Leukemia 26: 1576-1583, 2012.

37. Bailet O, Fenouille N, Abbe P, Robert G, Rocchi S, Gonthier N, Denoyelle C, Ticchioni M, Ortonne JP, Ballotti R, et al: Spleen tyrosine kinase functions as a tumor suppressor in melanoma cells by inducing senescence-like growth arrest. Cancer Res 69: 2748-2756, 2009.
38. Sung YM, Xu X, Sun J, Mueller D, Sentissi K, Johnson P, Urbach E, Seillier-Moiseiwitsch F, Johnson MD and Mueller SC: Tumor suppressor function of Syk in human MCF10A in vitro and normal mouse mammary epithelium in vivo. PLoS One 4: e7445, 2009.

39. Larive RM, Urbach S, Poncet J, Jouin P, Mascré G, Sahuquet A, Mangeat PH, Coopman PJ and Bettache N: Phosphoproteomic analysis of Syk kinase signaling in human cancer cells reveals its role in cell-cell adhesion. Oncogene 28: 2337-2347, 2009. 